\title{
Application of Grid-Level Battery Energy Storage System to Wind Power Fluctuation Smoothing
}

\author{
Tsai-Hsiang Chen, Rih-Neng Liao, Chun-Yen Yang, and Yen-Hsun Chiang
}

\begin{abstract}
Fluctuations in the output power of large-scale wind farms can have a considerable influence on power quality and stability, leading to the risk of grid frequency deviation, particularly in isolated power system. Fluctuations in wind power can be smoothing through the inclusion of wind-storage system. In this system, wind turbines are connected with battery energy storage system (BESS). This paper proposes a novel operation control strategy, referred to as prediction-based control (PBC). The proposed strategy (which utilizes predicted changes in wind power generation) can effectively smooth the fluctuation in the output power of wind farms (WFs), based on the concept of confidence intervals. Furthermore, the rolling-wave planning and dynamic control of the BESS enhances its capacity. Finally, the proposed control strategy provides an avenue by which to examine the characteristics of large-scale WFs and BESS for better planning, design, and operations.
\end{abstract}

Index Terms - Grid-level battery energy storage system, wind farm, real-time control, prediction-based control, wind turbines, wind power fluctuation.

\section{INTRODUCTION}

Growing demand for energy and alternative sources of electricity prompted the establishment of large-scale WFs in Taiwan, which are connected to and operate in parallel with the public grid. However, wind power is characterized by random fluctuations [1]-[3], and the fluctuation in output power from WFs brings enormous challenges to grid security, such as peak power and frequency deviation, stability, and power quality [4], [5]. Therefore, smoothing the fluctuation in output power of a WF is a critical issue.

A number of schemes have been developed with the aim of smoothing the fluctuations inherent in wind power generation, most of which employ energy storage systems with real-time control [6]-[8]. Unfortunately, most existing methods consider only present fluctuations in the power output of wind-storage systems, and not future fluctuations. Thus, in [9], it was indicated that BESS devices can be used to adjust the output active- and reactive-power and enable rapid response in compensating for power imbalances associated with fluctuations in wind power.

This paper proposes a novel operation control strategy, referred to as prediction-based control, for use in BESS systems to smooth the fluctuations in output power of WFs. The proposed prediction-based control system takes into

Manuscript received September 10, 2014; revised June 23, 2015.

Tsai-Hsiang Chen, Rih-Neng Liao, Chun-Yen Yang, and Yen-Hsun Chiang are with the Department of Electrical Engineering, National Taiwan University of Science and Technology, 43, Keelung Road, Section 4, Taipei, Taiwan (10607), R.O.C. (e-mail: thchen@mail.ntust.edu.tw). account anticipated fluctuations in the power generated by the WF (via forecasting) as well as the charging and discharging capability of BESS based on simple control strategy. In other words, prediction-based control method be able to facilitate dynamic operations in BESS systems. The efficacy of the proposed PBC method was demonstrated in a number of practical case studies.

\section{SYSTEM DESCRIPTION}

\section{A. System Configuration}

Fig. 1 illustrates a sample wind-storage system comprising a WF and BESS. The proposed prediction-based control is used to control the charging and discharging of the BESS system and coordinate the time-varying fluctuations in power output of the WF. The objective is to smooth the output power of the combined WF and BESS within an allowable range The output power of the wind-storage system is represented as follows:

$$
P_{s y s}(t)=P_{\mathrm{WF}}(t)+P_{\mathrm{BESS}}(t)
$$

where $P_{s y s}(t)$ represents the output power of the wind-storage system at time interval $t ; P_{\mathrm{WF}}(t)$ refers to the output power of the WF at time interval $t ; P_{\text {BESS }}(t)$ denotes the output power of the BESS at time interval $t$.

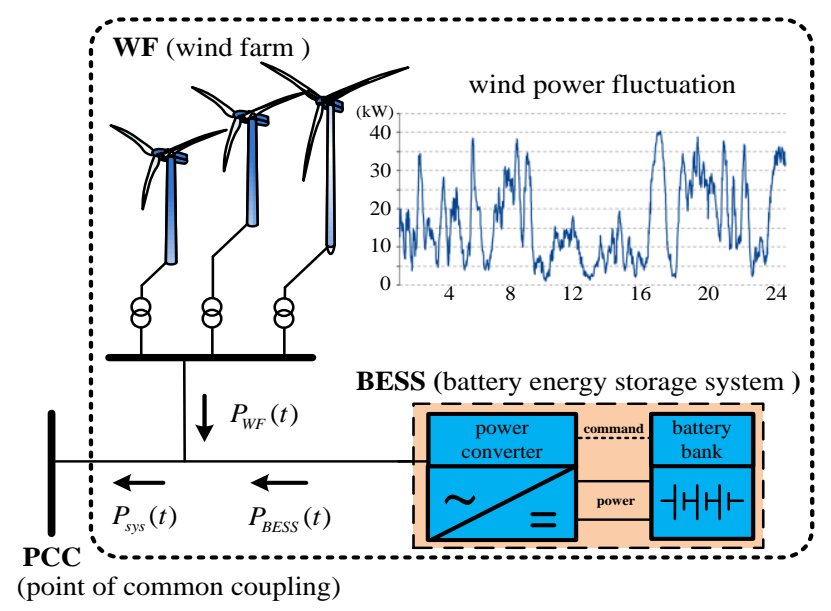

Fig. 1. Schematic diagram of the sample wind-storage system.

\section{B. Wind Farm}

The output power of a WF is primarily affected by its power characteristic curve, wind speed distribution (e.g. shape- and scale-parameters) and height of the turbine towers (e.g. measure and terrain). It is assumed that the WF consists 
of identical wind turbines, and that wind conditions are the same for all wind turbines.

\section{Battery Energy Storage System}

A BESS is characterized by its energy capacity, charge/discharge efficiency, charge/discharge power rate, etc. In order to protect the batteries form over-charging or -discharging, standard procedures related to battery management system (BMS) is required.

\section{WIND POWER GENERATION}

For fluctuations of large-scale wind farms that is necessary to consider the produced power in the daily operation planning of the power system. The first step is then to use forecasts of wind power. Nowadays, wind speed forecasting is still imprecise such that extending the prediction period leads to lower accuracy. The confidence interval $T_{0}$ is defined as a time interval that wind speed predictions can attain accuracy of $100 \%$, thus the reserves have to be kept for un-forecasted wind speed changes. In other words, forecasting error within the confidence interval can be disregarded. In this paper, wind speeds are forecast using the following process:

- Step 1: Express the wind speed as a Weibull distribution.

- Step 2: Obtain the shape and scale parameters by moment method with actual data related to wind speeds at the WF.

- Step 3: Measure wind speeds at an appropriate location and height.

- Step 4: Determine the wind power output by parabolic law.

\section{Prediction-Based Control Strategy}

Fluctuations in the output power of a WF is common due to variance in wind speed, turbulence intensity, tower shadow effect, and blade pitch, as well as the switching operations of the wind turbine. Many interconnection codes have requirements on the output power fluctuation regarding the network topology and load characteristics.

The resulting fluctuation in output power of the wind-storage system can be written as follows:

$$
\Delta P_{s y s}(t)=P_{s y s}(t)-P_{s y s}(t-\Delta t)
$$

Substituting Eq. 2 into Eq. 1 results in the following:

$$
\Delta P_{s y s}(t)=\Delta P_{W F}(t)+P_{B E S S}(t)
$$

Eq. 4 indicates that the control strategy of the BESS to maintain the output power fluctuation within the allowable maximum power fluctuation.

$$
\left|\Delta P_{s y s}(t)\right|<\Delta P_{M a x}
$$

Computing fluctuations in the output power of the WF, a sum of squared deviations fluctuations in the WF output power $\left(W F O P_{\text {sosd }}\right)$ was defined, as follows:

$$
W F O P_{\text {sosd }}=\sum_{t=1}^{T}\left(\Delta P_{\text {sys }}(t)\right)^{2}
$$

where $\Delta P_{s y s}(t)$ denotes fluctuations in the output power of the wind-storage system at time interval $t ; \Delta P_{M a x}$ represents the specified maximum allowable fluctuation in output power of the wind-storage system.

The maximum allowable fluctuation in output power specified according to the specific conditions and requirements of the grid to which the system is connected. Some constraints of the BESS should be complied, such as output power and SOC. For the wind-storage system, the target fluctuations in output power are less than the maximum allowable fluctuations, $\Delta P_{M a x}$. If $\Delta P_{s y s}(t)>\Delta P_{M a x}$, then the BESS must charge or discharge the battery bank in order to smooth fluctuations in output power of WF, otherwise, $P_{B E S S}(t)$ remains zero (i.e., the SOC of the battery bank will maintain the same state as that in time interval $t-\Delta t$ ). Due to the real-time control strategy of the BESS, the system is dependent on the instant SOC of the battery bank. This control strategy is therefore limited in its flexibility. For example, if the fluctuations in output power of WF exceed $\Delta P_{M a x}$ while the SOC of the battery bank is located at the minimum or the maximum values, then the BESS is unable to perform charge or discharge actions in compensation for the fluctuations smoothing. In this paper, a prediction-based control method was proposed to control the charging and discharging of the BESS to enhance the capacity utilization. BESS charging and discharging is controlled by the predicted changes in wind power generation.

\section{RESULTS AND DISCUSSION}

A wind-storage system consisting of a WF and BESS was used as a sample system to demonstrate the effectiveness of the proposed control strategy in smoothing fluctuations in

\begin{tabular}{|c|c|c|}
\hline \multirow{6}{*}{$\begin{array}{c}\text { WTGs } \\
\& \\
\text { WF }\end{array}$} & Brand model & ENERCON-E40 \\
\hline & Rated power & $0.6 \mathrm{MW}$ \\
\hline & Cut-in speed & $2.5 \mathrm{~m} / \mathrm{s}$ \\
\hline & Rated speed & $13 \mathrm{~m} / \mathrm{s}$ \\
\hline & Cut-out speed & $25 \mathrm{~m} / \mathrm{s}$ \\
\hline & Hub height & $46 \mathrm{~m}$ \\
\hline \multirow{5}{*}{ BESS } & Cell type: & Lithium-Ion \\
\hline & Rated capacity: & $1.5 \mathrm{MWh}$ \\
\hline & SOC range: & $10-95 \%$ \\
\hline & Power rateing: & $1 \mathrm{C}$ \\
\hline & Discharging/Charging efficiency: & 0.9 \\
\hline
\end{tabular}
output power. Critical specifications of the WF and BESS are presented in Table I.

TABLE I: CRITICAL SPECIFICATIONS OF THE WF AND BESS

Simulations of the proposed wind-storage system were conducted for all four seasons. The time intervals and prediction periods used in the simulations were set at 15 minutes and 1 hour, respectively. The maximum allowable 
fluctuation in output power was assumed to be $2 \mathrm{MW}$ and the control interval of the BESS was synchronized with that of the WF. Fig. 2-Fig. 4 present simulation results of the proposed wind-storage system with given local loads in a typical weekday in spring. The results include the output power of the WF, SOC of the BESS, and fluctuations in the output power of the WF.

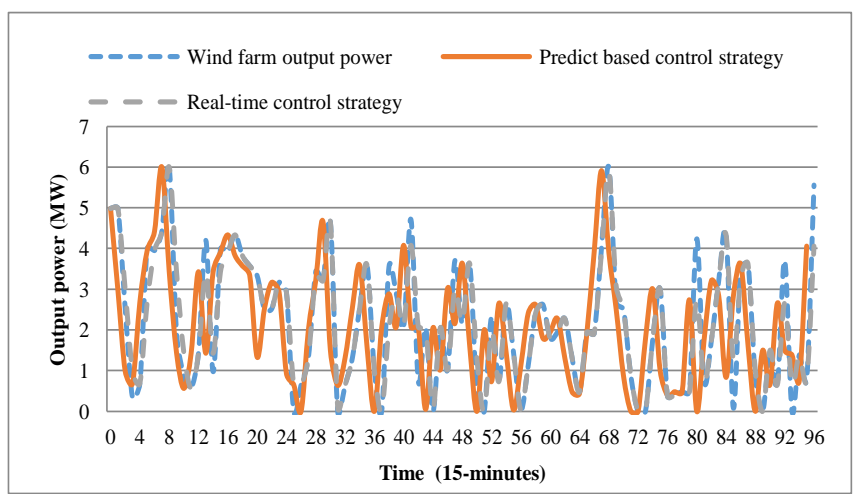

Fig. 2. Simulation results of output power of the sample wind-storage system for a typical weekday in spring.

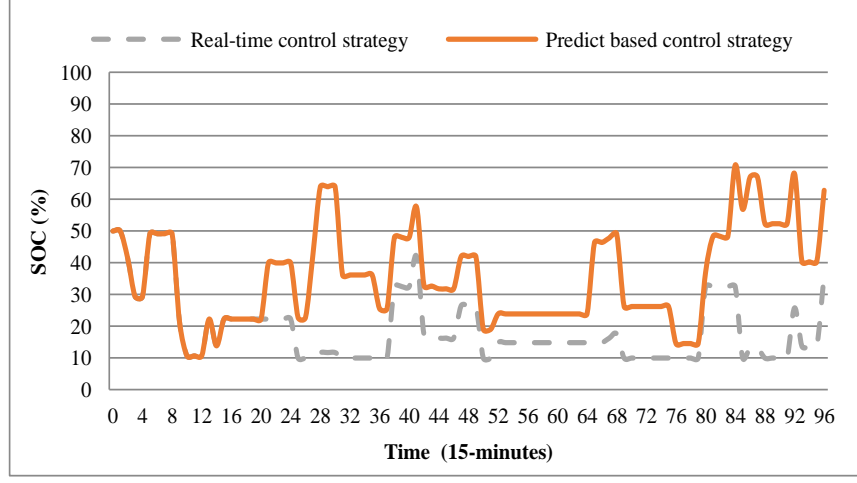

Fig. 3. Simulation results of SOC of the BESS for a typical week day in spring.

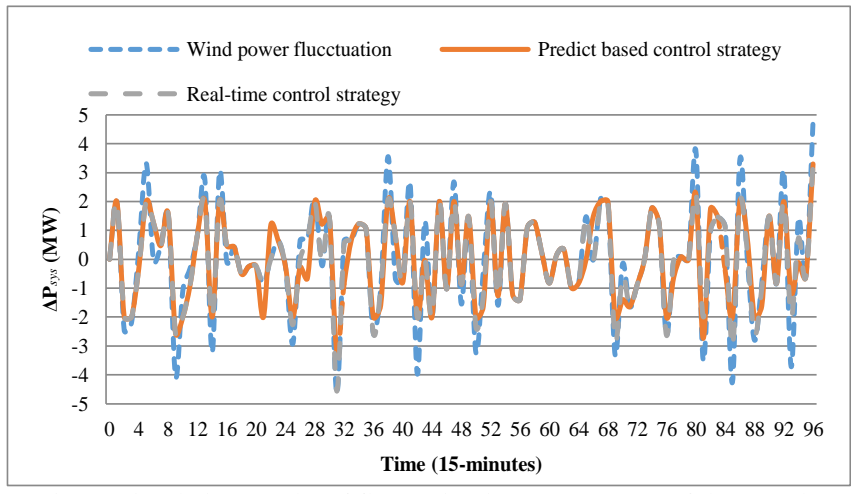

Fig. 4. Simulation results of fluctuation in output power of the sample wind-storage system for a typical weekday in spring.

Average seasonal wind speeds were determined for all four seasons: Spring (Mar., Apr., May), Summer (Jun., Jul., Aug.), Fall (Sep., Oct., Nov.) and Winter (Dec., Jan., Feb.), based on data from an actual WF in Taiwan. Simulations were carried out by the typical average date for each season. The output power fluctuations of the sample system and the probability of output power fluctuations violating the specified output power fluctuation limit for four seasons in a year are shown in Fig. 5, Fig. 6, respectively. Simulation results demonstrate the efficacy of the proposed system in smoothing fluctuations in the output power of the WF and thereby reducing the probability of violating the established limits. Our simulation results also show that the proposed predict based control strategy is superior to real-time control strategies in dealing with fluctuations in output power.

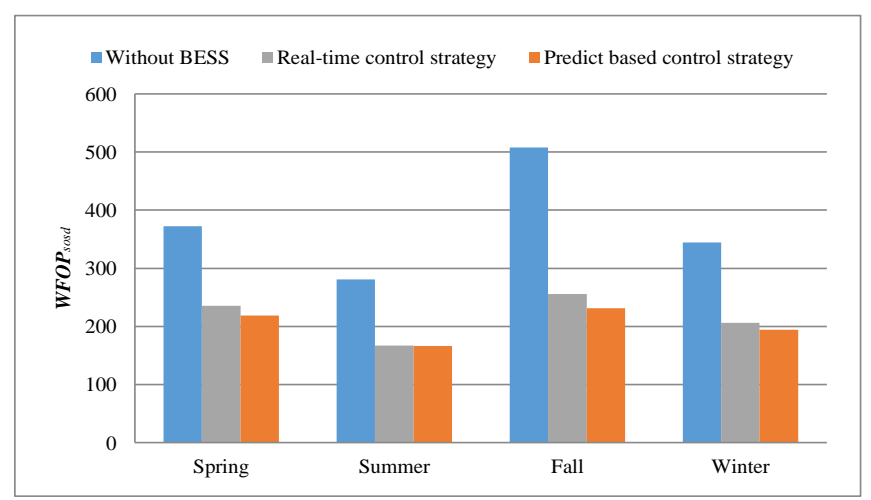

Fig. 5. Comparison of fluctuations in output power of the sample wind-storage system for each of the seasons.

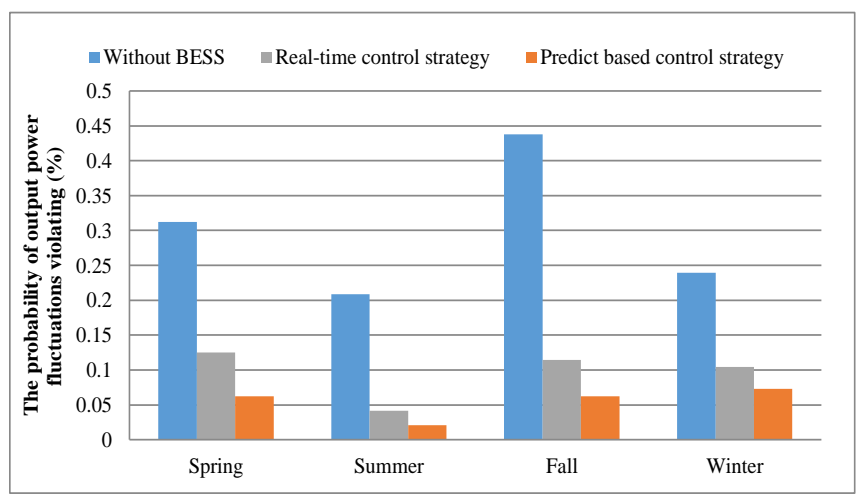

Fig. 6. Probability of fluctuations in output power violating the specified limits.

\section{CONCLUSION}

This paper evaluated the effectiveness of a BESS with prediction-based control for smoothing fluctuations in output power of a WF. The proposed prediction-based control was compared with real-time control methods, with regard to output power with and without a BESS, SOC for charging and discharging, and fluctuations in the output power of the wind-storage system under local load conditions. Simulation results demonstrate that if no measure was taken to deal with these fluctuations could have adverse effects on the lifespan of the BESS.

\section{ACKNOWLEDGEMENTS}

The authors would like to thank the Ministry of Science and Technology of the Republic of China, for financially supporting this research under Contract (No. MOST 103-2221-E-011-079-MY2).

\section{REFERENCES}

[1] X. Y. Yang and S. Y. Chen, "Wind speed and generated power forecasting in wind farm," Proceedings of the CSEE, vol. 25, pp. 1-5, 2005.

[2] X. C. Wang, P Guoc, and X. B. Huang, "A review of wind power forecasting models," The Proceedings of ICSGCE Technologies, vol. 12, pp. 770-778, 2011. 
[3] L. Ma, S. Luan, C. Jiang, H. Liu, and Y. Zhang, "A review on the forecasting of wind speed and generated power," Renewable and Sustainable Energy Reviews, vol. 13, pp. 915-920, 2009.

[4] S. Tao, W. Wang, H. Dai et al., "Voltage fluctuation and flicker caused by wind power generation," Power System Technology, vol. 27, pp. 62-66, 2003.

[5] E. Istvan, K. Jörg, F. Jens, M. E. Stephan, and W. Holger, "Modeling of wind turbines based on doubly-fed induction generators for power system stability studies," IEEE Trans. on Power Systems, vol. 22 , pp. 909-919, 2007.

[6] X. M. Yuan, S. J. Cheng, and J. Y. Wen, "Prospects analysis of energy storage application in grid integration of large-scale wind power," Automation of Electric Power Systems, vol. 37, pp. 14-18, 2013.

[7] J. Xie, L. U. Jiming, C. Mao, D. Wang, X. Fu, and W. Lin, "Optimal control of battery energy storage system based on variable smoothing time constant," Automation of Electric Power Systems, vol. 37, pp. 96-101, 2013.

[8] B. Hans and D. N. José, "A probabilistic method for energy storage sizing based on wind power forecast uncertainty," IEEE Trans. on Power Systems, vol. 26, pp. 1651-1658, 2011.

[9] V. P. Jukka and D. L. Peter, "Effect of energy storage on variations in wind power," Wind Energy, vol. 8, pp. 421-441, 2005.

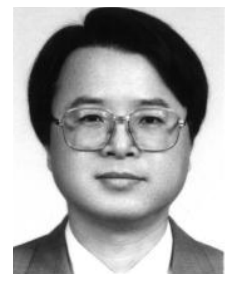

Tsai-Hsiang Chen was born in Taiwan, R.O.C., on March 15, 1953. He received his B.S. and M.S. degrees in electrical engineering from National Taiwan University of Science and Technology, Taipei, Taiwan, in 1980 and 1982, respectively. He received a $\mathrm{Ph} . \mathrm{D}$. degree in electrical engineering from the University of Texas at Arlington, Arlington, Texas, in 1990. Since 1982, he has been a faculty member of National Taiwan University of Science and Technology, and now he is a professor of electrical engineering and the director of Building Energy Efficiency and Renewable Energy Center. At present, his research interests include power system modeling and simulation, energy conservation, and micro-grid.

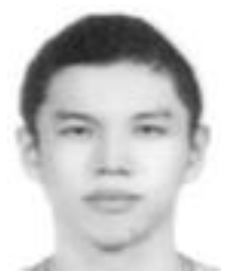

Rih-Neng Liao was born in Taiwan, the Republic of China, on August 7, 1985. He received his B.S., M.S degrees in electrical engineering from National Taiwan University of Science and Technology, Taiwan, the Republic of China, in 2007 and 2010, respectively. Now he is working for his $\mathrm{Ph} . \mathrm{D}$. degree at National Taiwan University of Science and Technology. His research interests include power system modeling and simulation, wind power system operation and analysis, interconnection evaluation studies of distributed generations, railway traction system, energy management system and electric vehicle. 
Bioenergy 
